\title{
A REPRESENTAÇÃO DE DOMÍNIOS DE CONHECIMENTO E UMA TEORIA DE REPRESENTAÇÃO: A ONTOLOGIA DE FUNDAMENTAÇÃO
}

\section{LA REPRESENTACIÓN DE DOMINIOS DE CONOCIMIENTOS Y UMA TEORÍA DE LA REPRESENTACIÓN LA ONTOLOGÍA DE FUNDACIÓN}

\author{
Maria Luiza de Almeida Campos - maria.almeida@pq.cnpq.br \\ Universidade Federal Fluminense - PPGCI/UFF. \\ Linair Maria Campos - linair@cisi.coppe.ufrj.br \\ Universidade Federal do Rio de Janeiro - CISI/COPPE/UFRJ. \\ Jackson da Silva Medeiros - jacksonmedeiros@yahoo.com.br \\ Universidade Federal do Rio Grande do Sul - PPGCOM/UFRGS.
}

\begin{abstract}
Resumo
A necessidade de disponibilizar informação de forma não ambígua e eficaz tem motivado o uso crescente de ontologias, cujo formalismo possibilita maior precisão em modelos de representação da informação. Nesse cenário, as ontologias de fundamentação possuem papel de destaque, ao definirem formalmente conceitos independentes de domínio, e fornecem uma base teórica sólida para embasar a construção de vocabulários específicos de cada domínio. A compreensão das noções formalizadas nas ontologias de fundamentação de base filosófica deve ser apropriada pelo cientista da informação, de modo a capacitá-lo para atuar nesse novo cenário, no qual a representação da informação deve estar voltada não só para o entendimento humano, mas também para o seu tratamento computacional, com o uso de inferências. O objetivo deste trabalho é apresentar algumas dessas noções, contidas na ontologia de fundamentação UFO-A, e ilustrar com um exemplo o diferencial semântico de uma modelagem embasada por essa ontologia em contraste com o que poderia ser representado utilizando-se um tesauro.
\end{abstract}

\section{Palavras-chave}

Ontologias. Ontologia de fundamentação. Representação de conhecimento

INTRODUÇÃO 
No escopo temático da organização, tratamento e da recuperação da informação o conceito de representação se insere. Este conceito nos coloca frente a indagações do tipo: Como re-apresentar ${ }^{1}$ um dado domínio ou uma dada informação contida em documentos? Como adaptar modelos de representação vigentes (esquemas de classificação, linguagens documentárias) a realidades diversas, com conteúdos por vezes complexos? Ou ainda, como re-apresentar/ elaborar modelos para uma dada organização física e/ou intelectual de conteúdos informacionais, por vezes vastos, com vistas à recuperação eficiente da informação? A representação é um conceito complexo, como também são complexas as soluções para ações de pesquisa, ensino e aprendizagem.

Tradicionalmente, a Ciência da Informação nasce já preocupada com o processamento semântico de informações por computadores. Saracevic (1995), discutindo a constituição da Ciência da Informação, afirma ser ela uma disciplina indissociavelmente ligada às tecnologias da informação. As décadas de 50 e 60 marcam as primeiras tentativas de usar o computador para processar conteúdos; são dessa época as experiências de pioneiros da Ciência da Informação como as de Shera (1957) com relação aos "semantic codes", e Luhn (1960), no sentido de viabilizar o processamento semântico de informações por computador. (MARCONDES; CAMPOS, 2008)

Uma de suas bases é a especificação semântica de relações. Relações estão na base do potencial dos computadores realizarem inferências. Esse termo deve ser entendido aqui não de forma literal, como uma atividade humana altamente sofistica e criativa, mas simplesmente a capacidade de um programa obter novas informações a partir de informações dadas, ou seja, relacionadas. A questão das relações é também explorada pela Ciência da Informação há tempos (FARRADANE, 1980; BROOKES, 1980; PERRAULT, 1994; KHOO, 2007; SALES, 2006; MARCONDES; MENDONÇA; MALHEIROS, 2006). Além disso, as relações são de fundamental importância para as atividades de reuso em ontologias (CAMPOS, 2007). A estes saberes se somam os relativos à modelização de domínios específicos, que possuem uma linha discursiva direta com os estudos das relações conceituais, onde a Ciência da Informação traz contribuições significativas como "sense making", de Dervin (1983), de Hjörland (2002), relativos a metodologias que poderiam ser usadas para aquisição de conhecimentos, na proposta de categorias que ajudem a estruturar de forma consistente esses domínios, como em Ranganathan (1967) e Dahlberg (1978a, 1978b). Ao longo de sua trajetória, a Ciência da Informação desenvolveu um arcabouço teórico e metodológico 
consubstanciado em obras como as de Otlet (1989), Ranganathan (1967), Dahlberg (1978a), Hjørland (2002) e do CRG - Classification Research Group (WILSON, 1972), entre outros, que potencialmente têm grande aplicabilidade no desenvolvimento de ontologias (CAMPOS, 2005).

No âmbito deste artigo, pretendemos ampliar tais discussões para o domínio dos estudos de representação e organização de modelos classificatórios e complementa-las com questões que vêm surgindo no âmbito dos estudos sobre "Ontologias de Fundamentação" (GUIZZARDI, 2005) que visam identificar o compromisso ontológico existente em modelos representacionais.

Compromisso ontológico, no contexto do presente trabalho, pode ser definido como um acordo firmado por uma comunidade sobre o significado que esta estabelece e é expressa em uma ontologia, tanto do ponto de vista da compreensão pelo homem quanto do tratamento pela máquina, através dos agentes de software. Isso implica em definir o vocabulário de uma forma que venha a minimizar ambigüidades, de modo que seu uso possa ser partilhado para representar e recuperar conhecimento entre comunidades afins, que se proponham a estar comprometidas com o acordo adotado. (NODINE; FOWLER, 2002).

As Ontologias de Fundamentação detêm forte fundamentação da Filosofia permitindo que a estrutura real de um domínio, seu compromisso ontológico, seja representada de forma fiel, clara e consistente. Isto permite que a representação realizada detenha uma semântica baseada no mundo real, restringindo interpretações sobre seus conceitos. Deste modo, as ontologias de fundamentação permitem que a construção de uma teoria sobre o domínio possibilite testar e validar um modelo conceitual. Assim, ao diferenciar os tipos de elementos que compõem um domínio e permitir sua representação, a ontologia de fundamentação explicita conceitos a partir de sua tipologia, estabelecendo sua posição em uma cadeia de elementos. Isto permite, a partir da noção de cada tipo de elemento, que a estruturação do domínio seja construída de forma a evitar proposições errôneas, aferindo qualidade à modelagem conceitual realizada e respectiva a representação do conhecimento em um domínio.

\section{ONTOLOGIA DE FUNDAMENTAÇÃO E SEU POTENCIAL SEMÂNTICO}

O termo ontologia tem origem no início século XVII, mais especificamente nos anos de 1606 e 1613, quando Jacob Lorhard escreve o Ogdoas Scholastica e Rudolf Göckel o 
Lexicon philosophicum. No entanto, ganha popularidade no âmbito da Filosofia através de Christian Wolff, com a publicação de Philosophia prima sive Ontologia (GUIZZARDI, 2005). Em Inteligência Artificial este termo é utilizado por empréstimo da Filosofia, sendo que estes estudos tiveram início nos anos 1990. A idéia era que ontologia fosse utilizada como possibilidade de representar a existência dos objetos e eventos, bem como suas relações. Neste sentido, ontologia era um artefato tecnológico que permitiria um modo de trabalhar com integração e recuperação da informação, gestão do conhecimento, sistemas multiagentes, etc. As ontologias pretendem que a comunicação possa se dar entre humanos e máquinas, diferentemente dos tesauros, por exemplo. Outro aspecto também muito ressaltado pela Inteligência Artificial foi o uso de ontologias para representar conhecimento em dado domínio, como relatado por Guarino (1998a). Uma ontologia é, segundo Guarino (1998b),

[...] uma teoria lógica correspondente ao significado pretendido de um vocabulário formal, ou seja, seu compromisso ontológico com uma conceituação particular de mundo. Os modelos pretendidos de uma linguagem lógica usada como vocabulário são restritos pelo seu compromisso ontológico. Uma ontologia indiretamente reflete esse compromisso (e a conceituação subjacente) pela aproximação desses modelos pretendidos [...]. (GUARINO, 1998b).

A partir de Guarino (1998a), podemos compreender uma ontologia como um artefato tecnológico que contém um conjunto de regras que delimitam o significado intensional de um vocabulário formal, permitindo que, a partir de um acordo ontológico (compromisso ontológico), conhecimento possa ser compartilhado e inferido.

No que se refere à sua constituição, segundo Ramalho (2010), uma ontologia é composta por: Classes e subclasses: agrupam um conjunto de elementos, coisas, do mundo real, que são representadas e categorizadas; Propriedades: descrevem as características e/ou qualidades das classes; Relacionamentos: ligações entre classes, descrevendo e rotulando os tipos de relações existentes no domínio representado; Regras e axiomas: enunciados lógicos que possibilitam impor condições e possibilitam a realização de inferências automáticas a partir de informações que não necessariamente foram explicitadas no domínio; Instâncias: indicam os valores das classes e subclasses, constituindo uma representação de objetos ou indivíduos pertencentes ao domínio modelado, de acordo com as características das classes, relacionamentos e restrições definidas; Valores: atribuem valores concretos às propriedades, indicando os formatos e tipos de valores aceitos em cada classe.

Tal organização, como consta acima, permite que determinado domínio de conhecimento seja representado e que, por se constituir em um artefato tecnológico que 
realiza inferências com base em seu conteúdo, informações sejam recuperadas de forma mais eficaz.

Como os estudos sobre ontologia são realizados em vários domínios, ocorre a criação de instrumentos variados. É possível identificar, mesmo com a apresentação de propriedades distintas, uma tipologia bem definida de ontologias, já que existem características e componentes básicos que estão presentes em grande parte delas (ALMEIDA; BAX, 2003). Neste sentido, podemos, dentre outras tipologias, analisá-las quanto ao grau de formalismo e ao aspecto estrutural. Enquanto a primeira diz que uma ontologia pode ser (a) altamente informal, (b) semi-informal, (c) semi-formal e (d) rigorosamente formal (USCHOLD; GRUNINGER, 1996), a segunda pode ser classificada em (a) ontologia de alto nível, (b) ontologia de domínio, (c) ontologia de tarefa e (d) ontologia de aplicação (GUARINO, 1998a).

A partir da necessidade de compor a representação de dado domínio, nosso interesse incide sobre a ontologia de domínio, a qual busca descrever formalmente classes de conceitos e os relacionamentos de determinada área com o objetivo de compartilhar um consenso terminológico. Além disso, sobre ontologia formal, a qual representa, formaliza o conhecimento existente, permitindo que seja acessado e compartilhado através de conceitos e categorias que satisfaçam a compreensão de um domínio. Deste modo, entendemos que uma ontologia formal está baseada em elementos como categorias e axiomas, demonstrando relações e propriedades dos conceitos e permitindo que a interpretação sobre dado conceito seja restrita, tomada através de um vocabulário controlado definido, delimitando o seu significado intensional e permitindo maior controle sobre o domínio que está sendo mapeado, assegurando qualidade às inferências realizadas.

A ontologia de fundamentação, por sua vez, tem por objetivo identificar categorias gerais de certos aspectos da realidade que não são específicos a um campo científico, descrevendo conhecimento independentemente de linguagem, de um estado particular das coisas ou ainda do estado de agentes (GUIZZARDI, 2005). Borgo e Masolo (2009) definem ontologias de fundamentação a partir de quatro itens: (i) têm grande alcance; (ii) podem ser altamente reutilizáveis em cenários de modelagem diferentes; (iii) são filosófica e conceitualmente bem fundamentadas; e (iv) são semanticamente transparentes e, portanto, ricamente axiomatizadas. 
Com base nas definições apresentadas, podemos dizer que uma ontologia de fundamentação é uma ontologia formal que permite a elaboração de modelos de domínios, sendo altamente reutilizável. É também caracterizada por ser filosoficamente bem fundamentada, recebendo aportes da linguística, ontologia formal e ciências cognitivas, permitindo a explicitação de uma visão da realidade, ou seja, do acordo ontológico estabelecido, com determinação de regras de restrição, bem como conceitos, categorias e meta-propriedades.

De acordo com Guizzardi (2005) e Guizzardi e outros (2008), a ontologia de fundamentação permite a explicitação dos compromissos ontológicos da ontologia que representa um domínio, agregando fidelidade, consistência e clareza na representação.

Percebemos, apoiados nos trabalhos de Guizzardi (2005), a principal contribuição das ontologias de fundamentação como sendo a busca pela caracterização fiel de uma conceituação, indo ao encontro do seu compromisso ontológico, permitindo que haja uma representação mais precisa do domínio em que a ontologia é empregada. Assim, fornecendo maior semântica e restringindo interpretações sobre seus conceitos com base em um vocabulário bem definido. Isto nos permite interpretar as ontologias de fundamentação como fornecedoras de uma estrutura ontológica, as quais criam um corpus capaz de validar o modelo conceitual gerado.

Uma vez que a ontologia de fundamentação é uma ontologia que pretende fornecer maior nível semântico do mundo para a modelagem conceitual de dado domínio de conhecimento, aborda questões como, por exemplo, (a) noções de tipos e suas instâncias; (b) objetos, e suas propriedades intrínsecas; (c) a relação entre identidade e classificação; (d) distinções entre tipos e suas relações; (e) relações parte-todo (GUIZZARDI, 2005), além de possuir elementos como classes, propriedades, relacionamentos, valores e regras na constituição do artefato.

Desta forma, o potencial semântico de uma dada ontologia é medido pelo modelo conceitual, e este está fundamentalmente relacionado com o compromisso ontológico passível de ser representado por este modelo, onde aspectos epistemológicos e ontológicos convivem. Nesta perspectiva, a abordagem epistemológica nos coloca diante de questões que visam responder sobre a natureza do conhecimento de um dado domínio, colocando o foco na discussão da relação entre conhecedor e o que pode ser conhecido. Já a abordagem ontológica pretende identificar qual é a forma e a natureza da realidade e, portanto, o que é que se pode saber sobre ela. Ambas as abordagens são temas de 
discussão desde os estudos filosóficos na Antiguidade Clássica, e também, como a literatura da Ciência da Informação tem evidenciado, estas questões já vem sendo tema de pesquisa de diversos estudiosos na atualidade (DOUSA, 2010; GNOLI; POLI, 2004).

A perspectiva epistemológica fornece bases teóricas para as ontologias, em especial as de fundamentação, e influenciam as escolhas que se encontram representadas nos conceitos nelas presentes:

Ontologia não é epistemologia, mas tem uma relação complexa com a epistemologia. Ontologia versa primariamente sobre as entidades, relações e propriedades do mundo, as categorias de coisas. Epistemologia versa sobre as entidades percebidas do mundo e as que se acreditam existir, suas relações e propriedades, i.e., maneiras de conhecer ou verificar coisas. (POLI; OBRST, 2009, p. 3).

Dessa forma, a perspectiva epistemológica é apoiada por crenças sobre a natureza da realidade e da verdade, que podem ser adotadas a partir de um acordo em um dado domínio, a partir de pressupostos ontológicos, ou seja, diferente da perspectiva epistemológica a perspectiva ontológica permitiria estabelecer quais classes de conceitos seriam admissíveis, suas relações e sua modelagem em relação a aspectos tais como existência no espaço e tempo.

\section{COMPROMISSO ONTOLÓGICO E SUA IMPORTÂNCIA NA FORMAÇÃO DE UMA CONCEITUALIZAÇÃO}

Guarino e Giaretta (1995) situam o papel do compromisso ontológico como o de um elemento fomentador da precisão entre a conceituação. Por conceituação definem, neste contexto, as regras implícitas, referentes ao entendimento humano de sua visão de mundo, como pensadas para serem utilizadas por um agente de software, a fim de tratar os objetos desse mundo e suas relações de interesse. O compromisso ontológico pode ser representado através de modelos conceituais, voltados para o entendimento humano, que refletem um método de raciocínio ou forma de pensar sobre uma dada realidade.

Assim, o estabelecimento de compromisso ontológico revela uma tomada de posição em relação aos objetos que se reconhecem como fundamentais em uma visão de mundo e que possibilitam o diálogo sobre esse mundo, e ainda em relação à maneira pela qual esses objetos são caracterizados. Vale ressaltar que existem diversos modos possíveis de um acordo sobre esta visão de mundo. Deve-se ter em mente, entretanto, que nas ontologias, principalmente nas chamadas ontologias de fundamentação, devido 
às restrições dos mecanismos de representação do conhecimento para as máquinas, a representação dos conceitos e de suas relações que constituem o seu modelo conceitual é sempre limitada e, portanto, aproximada.

Guarino situa o papel do compromisso ontológico como o de um elemento fomentador da precisão entre a conceituação e a representação de uma visão de mundo, esta última um modelo desse mundo, impreciso em algum grau em relação ao significado pretendido pelo homem. Essa imprecisão se dá devido ao fato de que as conceituações são entidades abstratas, que existem na mente de pessoas ou grupo de pessoas de uma comunidade (GUIZZARDI, 2007). Naturalmente, para que uma conceituação possa ser comunicada entre pessoas, esta deve ser expressa em termos de um artefato concreto que deve ser representado em uma linguagem, a qual não consegue ser suficientemente expressiva para representar a riqueza semântica da conceituação presente na mente humana.

Uma representação de conhecimento é um mecanismo usado para se raciocinar sobre o mundo, em vez de agir diretamente sobre ele. Nesse sentido, ela é, fundamentalmente, um substituto para aquilo que representa. [...]. Deve haver alguma forma de correspondência especificada entre o substituto e seu referente planejado no mundo. Esta correspondência é a semântica da representação. [...]. Fidelidade perfeita é, em geral, impossível, tanto na prática quanto em princípio. A única representação completamente precisa de um objeto é o objeto em si. Qualquer outra representação é imprecisa e, inevitavelmente, contém simplificações. (CAMPOS, 2004, p. 24).

O compromisso ontológico surge então para preencher a lacuna semântica entre o que o homem identifica nas suas conceituações de um mundo e o que é possível representar em uma ontologia através de uma linguagem. O compromisso se desdobra em dois: as coisas cuja existência se admite e o seu significado pretendido. As ontologias são uma forma de especificar para a máquina esse compromisso, através do uso de relações intensionais.

Desta forma, o compromisso ontológico vem ocupar o espaço de mediação entre a conceituação, que está no plano da semântica do entendimento humano e a ontologia, que está no plano do processamento pela máquina. É formado por um conjunto de regras que objetiva minimizar ambigüidades ou entendimentos errôneos dos conceitos do mundo sendo representado de acordo com a conceituação do homem, e embora seja voltado para o entendimento humano, é adequado para a conversão formal em uma linguagem que possa ser entendida pela máquina. Sendo assim, embora possa ser definido através 
de uma linguagem textual ou gráfica, o uso de uma linguagem formal auxilia na sua passagem para a linguagem que pode ser tratada computacionalmente.

A conceituação, ao contrário da ontologia, independe da linguagem utilizada e de um estado de coisas particular, existente ou não. Desse modo, é possível haver diferentes situações envolvendo os mesmos objetos, e sendo descritas por linguagens diferentes, porém envolvendo a mesma conceituação.

Assim, quanto maior o compromisso ontológico, ou seja, quanto mais estivermos restringindo as possibilidades de interpretação, maior a aproximação do modelo pretendido, por outro lado, menor é a possibilidade de esse modelo se encaixar em outras visões de mundo, e, portanto, menor a compatibilidade com esses outros modelos. Além disso, maior é a complexidade de sua atualização e maior é o custo de seu tratamento computacional. Por outro lado, se um compromisso ontológico de alto nível puder ser definido, a compatibilidade pode ser aumentada, na medida em que se passa a poder caracterizar de forma mais precisa a natureza dos elementos de um mundo:

[...] se nós queremos capturar a estrutura a priori dos indivíduos nós precisamos de um nível de detalhe suficiente para poder falar sobre a sua constituição interna, enquanto que para capturar a natureza dos indivíduos e suas relações nós também precisamos de categorias primitivas adequadas [...]. (GUARINO; CARRARA; GIARETTA, 1994, p. 2).

O compromisso ontológico pode ser representado através de modelos conceituais, voltados para o entendimento humano, que refletem um método de raciocínio ou forma de pensar sobre uma dada realidade, o qual se observa nos diferentes tipos de ontologia de domínios.

\section{ONTOLOGIA DE FUNDAMENTAÇÃO: A PROPOSTA DA UFO}

Em sua tese de doutorado, Guizzardi (2005) apresenta a construção de uma ontologia de fundamentação, denominada Unified Foundational Ontology (UFO), para o apoio a modelagem conceitual. Essa ontologia é dividida em três fragmentos: 1) UFO-A, é a ontologia de endurants ${ }^{2}$ (endurantes), que define o cerne da UFO (com termos como, por exemplo, tipos, relações todo-parte, espaço de valores de atributos, entre outros); 2) UFO-B define, adicionalmente à UFO-A, uma ontologia com termos relativos aos perdurants (ou perdurantes, como, por exemplo, estados, processos, eventos, relações temporais, entre outros.); e 3) UFO-C define, adicionalmente à UFO-B, termos

${ }^{2}$ Os conceitos de endurante e perdurante serão explicados mais adiante. 
relacionados a conceitos sociais (tais como plano, ação, objetivo, agente, intencionalidade, comprometimento e compromisso). A partir destes elementos que, em essência, constituem-se na divisão geral de categorias nestes fragmentos do modelo, buscam agrupar conceitos e categorias gerais que, apoiados por uma ontologia de fundamentação, subsidiam a modelagem conceitual.

Neste trabalho mostraremos especificamente a UFO-A, detalhada em Guizzardi (2005), sendo esta pautada em endurantes (também conhecidos como continuantes). Endurantes são entidades que não mudam sua essência ao longo do tempo, preservando sua identidade durante as mudanças que ocorrem durante o período de sua existência: "Você é hoje a mesma pessoa que você foi ontem" (GRENON; SMITH, 2004). Organismos, células e moléculas são exemplos de entidades cuja natureza é endurante.

Uma vez que a UFO-A é uma ontologia que pretende fornecer maior nível semântico do mundo para a modelagem conceitual de dado domínio de conhecimento, aborda questões como (a) noções de tipos e suas instâncias; (b) objetos, e suas propriedades intrínsecas; (c) a relação entre identidade e classificação; (d) distinções entre tipos e suas relações; (e) relações parte-todo (GUIZZARDI, 2005), além de classes, propriedades, relacionamentos e regras.

Antes, porém, é importante apresentar algumas noções filosóficas básicas como identidade, rigidez e dependência, visando melhor compreensão dos elementos da UFOA, uma vez que estas noções são de importância ímpar na identificação de entidades em um domínio.

\subsection{Noções filosóficas básicas aplicadas na UFO}

Identidade é a propriedade determinante do objeto, a especificação do que a coisa realmente é, ou seja, ao analisar duas entidades, as quais exibem propriedades diferenciadas, diz se estas podem ser consideradas como sendo as mesmas (CAMPOS, 2001). Neste sentido, Guarino (1998b), diz que a partir da utilização do critério de identidade como princípio ontológico para caracterizar as coisas, e a conseqüente limitação de conexões "é um", a taxonomia resultante reflete uma semântica clara.

A noção de rigidez diz que uma coisa é rígida quando ao longo do tempo é aplicável a todas as instâncias que dela derivam. Pode-se ilustrar isso a partir de duas propriedades: pessoa e estudante. A primeira é uma propriedade rígida, uma vez que uma pessoa nunca deixa de ser uma pessoa ao longo de sua existência, enquanto a 
propriedade estudante é não rígida, já que a propriedade estudante pode aparecer em apenas um período de tempo da existência de determinada entidade (GUIZZARDI, 2005).

A noção de dependência, como destaca Guarino (1998b), revela algumas questões, como: (i) a existência real de um individual implica necessariamente na existência real de outro indivíduo específico (dependência rígida), por exemplo, a relação existente entre uma pessoa e seu cérebro; (ii) a existência real de um indivíduo implica necessariamente na existência de algum indivíduo real que pertença a uma classe específica (dependência genérica), como, por exemplo, a relação entre uma pessoa e seu coração, e; (iii) o fato que um individual pertence a uma classe em particular necessariamente implica a existência de um individual diferente pertencente a outra classe (dependência de classe), sendo, neste caso, como exemplo, a existência de relacionamento entre a classe "Pai" e a classe "Filho".

\subsection{Elementos da UFO}

Devemos, inicialmente, esclarecer que a UFO-A faz um primeiro recorte entre os seus elementos distinguindo-os em universals e individuals. Universals são entidades que comportam um conjunto de características seguindo padrões gerais, agrupando diferentes individuals. Individuals, por sua vez, dizem respeito a coisas que existem e mantêm identidade única, ou seja, são as instâncias.

Universals e individuals que necessitam de outras entidades para existir são chamados moments. De modo inverso, universals e individuals que não dependem de outras entidades são conhecidos como substantials. Exemplos de moment individuals são: uma cor, uma carga elétrica e um sintoma e exemplos de substantial individuals são: uma mesa, uma pessoa, uma cadeira.

Existe ainda a diferença entre intrinsic moments e relational moments (relators). $O$ primeiro depende de um único individual, como uma cor, uma dor de cabeça. O segundo tem sua existência condicionada a existência de duas ou mais entidades, como, por exemplo, um casamento, um aperto de mão, um beijo.

Uma abordagem para modelagem da relação existente entre intrinsic moments e suas representações em estruturas cognitivas humanas é apresentada na teoria dos espaços conceituais, proposta por Gardenfors, sendo esta teoria baseada na noção de quality structure. A ideia é que, de modo geral, em vários moment universals perceptíveis ou imagináveis há uma estrutura de qualidade associada na cognição humana. Por exemplo, altura está associada a uma estrutura unidimensional com ponto zero e 
contagem não negativa. Outras propriedades, como cor e sabor, por exemplo, estão relacionadas a estruturas multidimensionais. Este ponto exato percebido pode ser representado em uma quality structure é nomeado um quale. Estas estruturas são exemplos de abstract particulars (moments) (GUIZZARDI, 2005).

Figura 1: Representação de Moments.

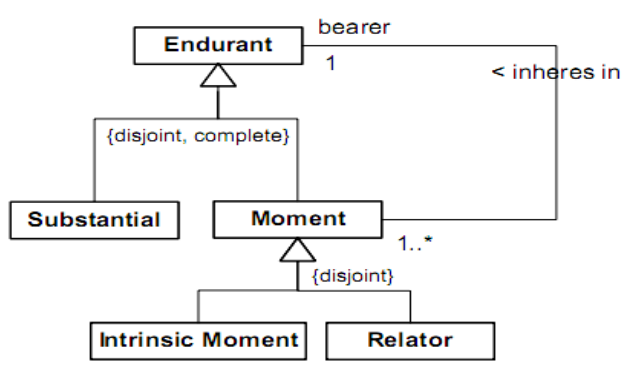

Fonte: Guizzardi (2005, p. 215)

Universals podem ser classificados em substancial universal ou moment universal. Um substantial, como já foi abordado acima, é uma entidade que mantém sua identidade no tempo, sendo existencialmente independente de qualquer outra entidade. Um moment, ao contrário, não é parte da essência do objeto, assim, pode apenas existir a partir da existência (dependência) de outra entidade. Os substantial universals podem ser classificados em sortal universals ou mixin universals.

Figura 2: Distinções ontológicas em uma tipologia de universais substanciais.

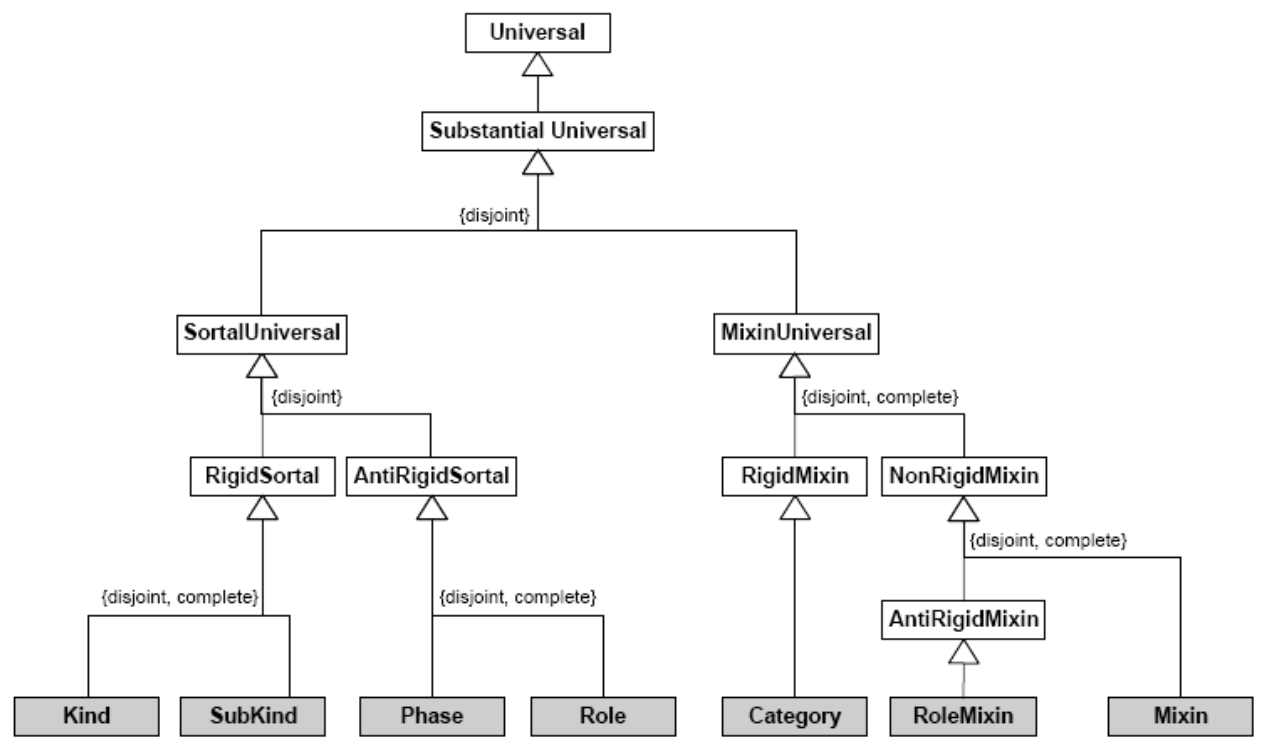

Fonte: Guizzardi, (2005, p. 106)

Sortal universal é uma entidade que carrega um princípio de identidade para suas instâncias, permitindo observar se duas entidades são as mesmas a partir de 
características fornecidas. Mixin universal, por sua vez, agrega conceitos de diferentes características e identidades. Com isso, entendemos que estes conceitos podem ser considerados classes que agregam entidades, ainda que de essência diferenciada (GUIZZARDI, 2005).

Neste ponto é importante rever a noção de rigidez, para então compreender antirigidez e não-rigidez. Em relação a um substancial universal, dizemos que ele é rígido quando uma entidade classificada dentro dele é uma instância durante todo tempo em que o substantial universal existir, independente do mundo a que é aplicado. Um substantial universal é anti-rígido se a entidade instanciada a ele puder deixar de existir ao longo de sua existência, não sendo aplicável a totalidade de instâncias do universal. Já o substantial universal não-rígido será aplicável a pelo menos uma de suas instâncias.

Kind representa um sortal substantial que fornece um princípio de identidade para suas instâncias, sendo responsáveis pela estruturação da taxonomia representante do domínio. Kinds podem ser especializados em outros subtipos rígidos que herdam o princípio de identidade e são chamados subkinds.

Figura 3: Exemplo de kind e subkind.

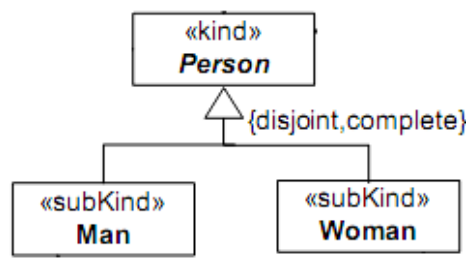

Fonte: Guizzardi (2005)

Phase e role são sortals anti-rígidos. Phases são constituídos de partes temporais ou são representados em determinado mundo, caracterizando-se, como o próprio termo indica, uma fase passageira de um universal. Role é um processo, função executada por uma entidade em determinado contexto ou por um período de tempo. É exemplo de phase a adolescência e de role ser empregado.

Figura 4: Exemplo da noção de phase.

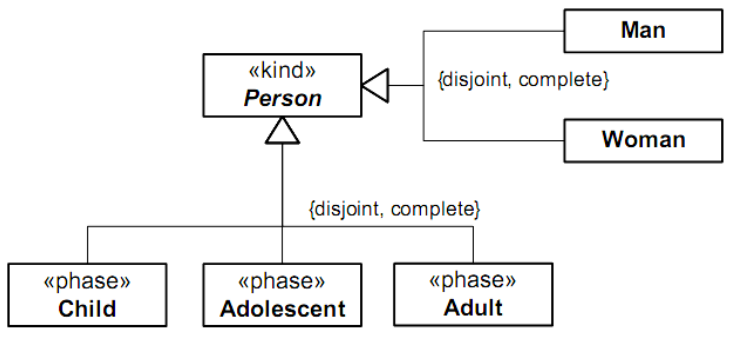


Fonte: Guizzardi (2005, p. 110)

Figura 5: Exemplo do sortal role.

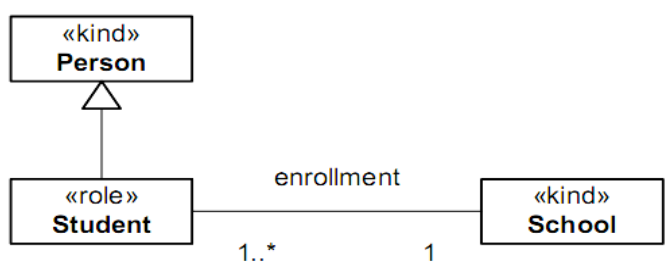

Fonte: Guizzardi (2005, p. 129)

Category é um rigid mixin. Engloba entidades de espécies diferentes, com características essenciais em comum. Não fornece um princípio de identidade.

Figura 6: Exemplo de category

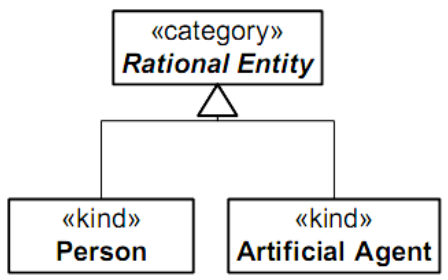

Fonte: Guizzardi (2005, p. 113)

Rolemixins são constituídos por propriedades comuns abstraídas de papeis. Um mixin agrega propriedades que são essenciais para algumas instâncias e acidentais para outras. Por exemplo, ter a propriedade de ser "sentável" é um mixin, visto que esta pode ser uma característica tanto de uma cadeira (para esta uma característica essencial) quanto de um engradado sólido (para este uma característica acidental).

Figura 7: Exemplo de Rolemixin.

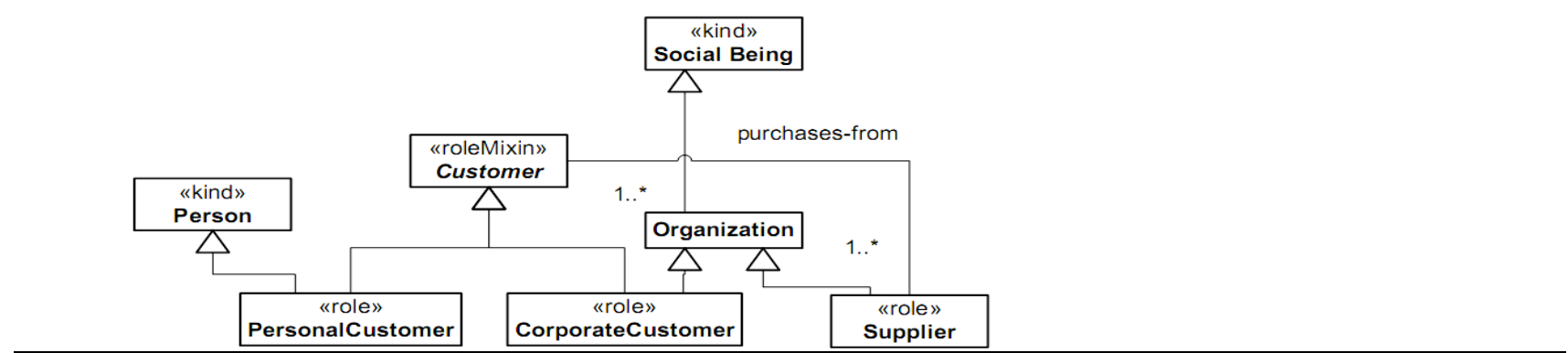


Fonte: Guizzardi (2005, p. 111)

Figura 8: Exemplo de mixin.

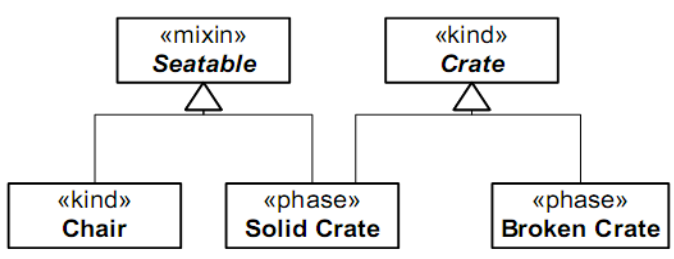

Fonte: Guizzardi (2005, p. 113)

$\mathrm{Na}$ UFO, as relações entre entidades são também consideradas entidades, podendo ocorrer de duas formas: relações formais (formal relations) e relações materiais (material relations). Nas relações formais a relação entre entidades é direta, ou seja, não possui intermediário, sendo representada pelas relações como instanciação, parte-todo, membros, associação, etc. Como relações formais podem ser incluídas as relações de comparação, como maior que, mais alto que, passando a se chamar relações formais comparativas. Já as relações materiais mantêm uma entidade (relator) como intermediária, sendo ele um individual que permite conectar entidades. Pode-se usar como exemplo "paciente recebe tratamento em uma unidade médica", onde "tratamento" é o relator, o intermediário da relação, podendo esta entidade compreender características adicionais que uma relação formal não comportaria, tal como duração do tratamento, por exemplo. Por conseqüência, pode-se compreender que o relator é dependente de duas ou mais entidades.

\subsection{Aplicações para ontologias de fundamentação}

Ontologias de fundamentação como a UFO têm sido usadas para apoiar a modelagem de conceitos para fins diversos. Estes vão desde a compreensão de requisitos em um modelo de negócio que é apoiado por um sistema informatizado (MARTINS et al., 2011), até a representação do recorte de um domínio para fins de descrição e recuperação de informação em contextos diversos, como, por exemplo, em sites de jornalismo (CAROLO; BURLAMAQUI, 2011). Tais iniciativas, que envolvem o uso 
de ontologias, têm tido um grande crescimento nos últimos anos (GUIZZARDI; FALBO; GUIZZARDI, 2008).

O que essas iniciativas têm em comum é a necessidade de minimizar entendimentos ambíguos e fornecer uma semântica e entendimento mais precisos não só dos conceitos representados, como também de suas naturezas e relações. Essa necessidade difere daquela das linguagens documentárias tais como os vocabulários controlados ou os tesauros, onde o foco é na recuperação de informação para pessoas, e onde a inferência de conhecimento não é considerada como requisito. Por exemplo, como no caso de um Tesauro do Folclore como instrumento de navegação e recuperação de informações digitais (sons, imagens) associados aos conceitos dessa temática.

Nesse sentido, é importante que o Profissional da Informação se aproprie dos novos métodos e conhecimentos necessários para atender às novas demandas que se fazem presentes, e que envolvem as noções filosóficas e epistemológicas contidas em ontologias de fundamentação como, por exemplo, a UFO. O entendimento dessas noções e as notações adotadas para a sua modelagem são complexos, mas oferecem um campo vasto de aplicação, do qual o Profissional da Informação não pode se omitir, sob pena de diminuir o papel da área em um cenário dinâmico que avança rapidamente.

Como forma de ajudar a materializar esses novos insumos, apresentaremos a seguir um exemplo simples e limitado, embora bastante ilustrativo, do poder semântico das ontologias de fundamentação na modelagem de um recorte de domínio.

\section{EXEMPLO DE APLICAÇÃO DA UFO PARA FUNDAMENTAR O DOMÍNIO DE FOLCLORE}

Utilizamos neste exemplo um termo do Tesauro de Folclore e Cultura Popular Brasileira do IPHAN. Este tesauro (doravante denominado Tesauro do Folclore) pode ser classificado como um tesauro conceitual, pois além de possuir parte alfabética e sistemática, ele agrega aos termos e suas relações definições conceituais referendadas por um grupo de especialistas do domínio.

De acordo com o Tesauro do Folclore, "São Pedro" é definido como: "Santo escolhido por Jesus Cristo para ser o apóstolo encarregado de seu rebanho; seu nome de batismo era Simão; filho de Jonas, era pescador e tinha uma frota de barcos pesqueiros, juntamente com seu irmão André, Tiago e João". O conceito possui um termo associado, 
denominado "Ciclo Junino", cuja definição é "Tempo que se inicia no dia 13 de junho, Dia de Santo Antonio, e termina com a festa de São Marçal, no dia 30 de junho [...]".

Ao representarmos o conceito de "São Pedro" de acordo com a definição do Tesauro do Folclore, com a fundamentação da UFO-A (Figura 9), mesmo que de modo simplificado, algumas questões podem ser trabalhadas de forma mais precisa. Por exemplo, Simão desempenha três papéis: santo, apóstolo e pescador. Se fosse o caso, poderíamos associar informações distintas a cada um desses papéis, de modo a separar o que fosse pertinente apenas a cada um deles. De fato, se quisermos saber sobre a vida do apóstolo Pedro, observa-se que ele foi escolhido por Jesus Cristo. Por outro lado, se quisermos saber sobre a vida do pescador Pedro, observa-se que ele possui barcos. Ainda assim, somos capazes de saber que são a mesma pessoa (relação mesmoQue), apesar de ser com papéis distintos. Dessa forma, se quisermos saber sobre os outros aspectos de sua vida, é só escolher o papel desejado. 
Figura 9: modelagem do conceito de São Pedro, fundamentado pela UFO-A.

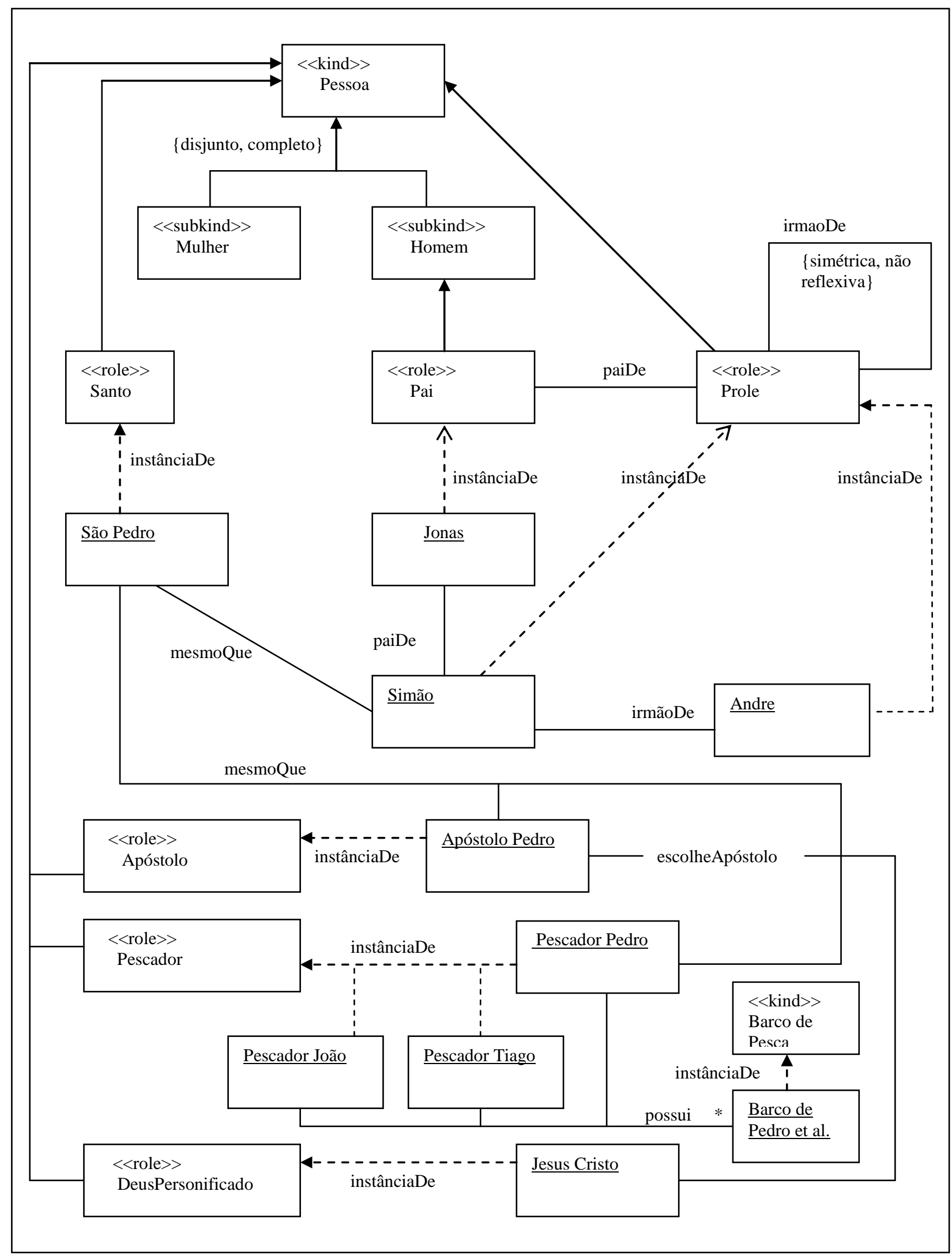


Outra questão, que surge é que nada é dito sobre a mãe de Simão. Dessa forma, Andre poderia ser apenas irmão de Pedro por parte de mãe, ou seja, de acordo com a definição do tesauro, não podemos afirmar que Jonas era pai de Andre. Pode ser que para o tesauro do folclore essa informação não seja relevante, mas poderia ser para outro domínio e, nesse caso, o modelo deixa isso bem claro.

Outra questão é a associação do conceito "São Pedro" com o de "Ciclo junino". Para representar que São Pedro de alguma forma está ligado ao ciclo junino, seria conveniente resgatar que o dia de São Pedro (29 de junho), que não consta da definição do conceito de "São Pedro", está dentro do intervalo nomeado como ciclo junino (13 de junho a 30 de junho), do contrário, não fica óbvio o porquê dessa associação. Por motivos de espaço não representamos essa ligação, que se daria entre o conceito de "Data comemorativa de São Pedro" (ou dia de São Pedro) e o do "Ciclo Junino". Entretanto, podemos adiantar que a modelagem evidenciaria a importância de associar a informação do dia de São Pedro na definição desse conceito, considerando que é importante estabelecer o seu vínculo com o conceito de "Ciclo Junino".

Outro conceito que sentimos necessidade de destacar é o de "Deus Personificado", uma vez que, do contrário, "Jesus Cristo" seria considerado uma pessoa comum. Nesse caso, a título de exemplo, inserimos esse conceito no modelo para destacar o papel diferenciado de Jesus Cristo. Naturalmente, o destaque desse papel não é obrigatório, depende do que é relevante para o domínio. Porém, ao modelar esse conceito ao lado de outros tais como o de "Apóstolo Pedro", "São Pedro" e "Pescador Pedro", intuitivamente sentimos necessidade de situar o papel de "Jesus Cristo" frente aos outros, de modo a entendê-lo como uma pessoa especial no contexto religioso que se apresenta.

Podemos observar ainda a riqueza semântica das relações no modelo. Por exemplo, ao criar o conceito de "Prole", podemos indicar que um pai pode ter uma prole e que essa prole estabelece uma relação entre si de irmãoDe. Essa relação é simétrica, ou seja, se "Simão" é irmão De "Andre", podemos inferir que "André" é irmãoDe "Simão". Como a relação é não reflexiva, podemos estar certos de que André não pode ser irmão de si mesmo, o que pode ser óbvio para a interpretação humana no caso de irmãos, mas que pode não ser óbvia para outro tipo de situação, como, por exemplo, entre a classe pessoa e a relação ensina. Dependendo do caso, podemos querer expressar o fato de que uma pessoa pode ensinar a si mesma (caso de autodidatismo), onde a reflexividade se aplica. Porém, também pode ser o caso de uma escola tradicional onde a pessoa de 
um professor ensina a pessoa de um aluno, e, nesse caso, a reflexividade não se aplica (ou seja, uma pessoa, seja professor ou aluno, não pode ensinar a si mesma).

No modelo acima, podemos indicar ainda que os subtipos "Homem" e "Mulher" são disjuntos e completos, deixando claro que essas subclasses são todas as possíveis divisões de "Pessoa" (por isso, diz-se completo) e também que se uma "Pessoa" é homem não pode ser mulher. Dessa forma, como podemos observar, um modelo que permita expressar esses tipos de restrições contribui para evitar entendimentos ambíguos. O modelo também permite expressar a cardinalidade das relações (que, para simplificar colocamos apenas na relação possui), como, por exemplo, para expressar que os pescadores Pedro, Tiago e João possuem mais de um barco (caracterizando, de forma simplificada, a noção de frota).

Outra vantagem da modelagem é deixar claras as afirmativas contidas na definição, de modo a evitar enganos de interpretação. Por exemplo, uma leitura apressada ou desatenta, poderia dar a impressão de que Tiago e João também são irmãos de Simão (o que estaria expresso se a redação fosse "... juntamente com seus irmãos" em vez de “... juntamente com seu irmão”). A modelagem também ajuda a evidenciar quais conceitos estão sendo considerados relevantes, o que auxilia a validar o recorte do domínio, expressado a partir da definição dos seus conceitos.

\section{CONCLUSÃO}

A necessidade de disponibilizar informações, muitas vezes de natureza complexa e variada, tem levado as instituições a valorizarem seus ativos informacionais, buscando organizá-los de forma mais eficiente, representando-os e articulando-os de forma mais precisa.

Nesse cenário, nos últimos anos, o uso de ontologias tem se intensificado, como apoio a iniciativas que demandam o uso de modelos de representação mais precisos e formais, de modo que minimize ambigüidades de entendimento e, ainda, que possua uma representação que permita ser tratada computacionalmente.

Até os últimos anos, antes do potencial de tratamento informacional imposto por este novo cenário, um dos papéis exercido pelo profissional de informação se restringia ao desenvolvimento de ferramentas semânticas capazes de representar um dado domínio de conhecimento (através de linguagens documentárias como tesauros, taxonomia e esquemas de classificação), possibilitando certa precisão e compatibilidade entre a 
linguagem utilizada para o tratamento informacional e a linguagem utilizada pelo usuário para a recuperação da informação.

No novo cenário, onde as ontologias formais se inserem, já é possível, de algum modo, não só a representação do conhecimento de um dado domínio, mas a derivação do conhecimento por sobre este domínio, através de uma semântica expressa em linguagens formais, possível de ser explorada computacionalmente para fins de inferência.

Desta forma, novas estratégias de conhecimento estão sendo requeridas, onde conteúdos diferenciados dos até agora estudados e pesquisados na área precisam de uma atualização sobre novos horizontes teóricos e metodológicos, sob pena de não ocuparmos o espaço neste novo cenário informacional.

\section{REFERÊNCIAS}

ALMEIDA, M. B.; BAX, M. P. Uma visão geral sobre ontologias: pesquisa sobre definições, tipos, aplicações, métodos de avaliação e de construção. Ciência da Informação, Brasília, v. 32, n. 3, p. 7-20, 2003.

BORGO, S. MASOLO, C. Foundational choices in DOLCE. In: STAAB, S.; STUDER, R. (Ed.). Handbook on Ontologies. 2. ed. Springer Verlag, 2009. p. 361-382. Disponível em: <http://www.loa-nr.it/Papers/lOShandDOLCEv16.pdf>. Acesso em: 22 de out. de 2011.

BROOKES, B. The foundations of information science - Part I. Philosophical aspects. Journal of Information Science, v. 2, p. 125-133, 1980.

CAMPOS, L. M. Diretrizes para definição de recorte de domínio no reúso de ontologias biomédicas: uma abordagem interdisciplinar baseada na análise do compromisso ontológico. 2011. Tese (Doutorado em Curso de Pós Graduação em Ciência da Informação) - UFF/IBICT, 2011.

CAMPOS, M. L. A. A organização de unidades de conhecimento em hiperdocumentos: um modelo conceitual como um espaço comunicacional para a realização da autoria. 2001. 186p. Tese (Doutorado em Ciência da Informação) - IBICT - UFRJ, 2001.

CAMPOS, M. L. A. INTEGRAÇÃO DE ONTOLOGIAS: O domínio da Bioinformática e a problemática da compatibilização terminológica. (Projeto de Pesquisa submetido ao CNPq no período de 2005 a 2008). Universidade Federal Fluminense- Departamento de Ciência da Informação, 2005.

CAMPOS, M. L. A. Integração de Ontologias: o domínio da bioinformática. RECIIS Revista de Comunicação, Informação \& Inovação em Saúde, v. 1, p. 117-121, 2007.

CAMPOS, M. L. A. Modelização de domínios de conhecimento: uma investigação de princípios fundamentais. Ciência da Informação, Brasília, v. 33, n. 1, p. 22-32, 2004. 
Disponível em: <http://revista.ibict.br/index.php/ciinf/article/view/56/53>. Acesso em: 22 de out. de 2011.

CAROLO, F.; BURLAMAQUI, L. Improving web content management with semantic technologies. Disponível em: <http://semtech2011.semanticweb.com/uploads/handouts/Carolo_Fernando_3919_3311.p df>. Acesso em: 02 de ago. de 2011.

DAHLBERG, I. A referent-oriented analytical concept theory of interconcept. International Classification, Frankfurt, v.5, n.3, p.142-150, 1978a.

DAHLBERG, I. Ontical structures and universal classification. Bangalore: Sarada Ranganathan Endowment, 1978b. 64p.

DERVIN, B. An overview of sense-making research: concepts, methods and results. Paper presented at the annual meeting of the International Communication Association. Dallas, TX. 1983.

DOUSA, T. M. The simple and the complex in E.C. Richardson's theory of classification: observations on an early $\mathrm{KO}$ model of the relationship between ontology and epistemology. In: Paradigms and conceptual systems in knowledge organization, p. 15-22, 2010.

FARRADANE, J. Relational indexing - Part I. Journal of Information Science, v. 1, p. 267276, 1980.

GRENON, P.; SMITH, B. SNAP and SPAN: Prolegomenon to geodynamic ontology. In: Spatial Cognition and Computation, 2004.

GUARINO, N. Formal ontology and information systems. In: FOIS '98, 1., 1998, Trento, Italy. Proceedings... Amsterdam: IOS Press; Tokyo, 1998a. p. 3-15.

GUARINO, N. Some Ontological Principles for Designing Upper Level Lexical Resources, In: First International Conference on Language Resources and Evaluation. Proceedings... Granada, Spain, May, 1998b.

GUARINO, N.; CARRARA, M.; GIARETTA, P. Formalizing ontological commitments. In: The Twelfth National Conference on Artificial Intelligence. Proceedings... v.1, p. 560-567, 1994.

GUARINO, N.; GIARETTA, P. Ontologies and knowledge bases: towards a terminological clarification. In: N. J. I. MARS (Ed). Towards very large knowledge bases: knowledge building and knowledge sharing. Amsterdam: IOS Press, 1995. p. 25-32.

GUIZZARDI, G. Ontological foundations for structural conceptual models. 416f. Tese (PhD em Computer Science) - Twente University of Technology, Twente, Holanda, 2005.

GUIZZARDI, G. On Ontology, ontologies, Conceptualizations, Modeling Languages, and (Meta) Models. In: VASILECAS, O.; EDLER, J.; CAPLINSKAS, A. (Org.). Frontiers in Artificial Intelligence and Applications, Databases and Information Systems IV. Amsterdã: IOS Press, 2007. 
GUIZZARDI, G.; FALBO, R.; GUIZZARDI, R. S. S . A importância de Ontologias de Fundamentação para a Engenharia de Ontologias de Domínio: o caso do domínio de Processos de Software. Revista IEEE América Latina, v. 6, n.3, p. 244-251, 2008.

GNOLI, C.; POLI, R. Levels of reality and levels of representation. Knowledge Organization, v. 31, n.3, p. 151-160, 2004.

HJØRLAND, B. Domain analysis in information science: eleven approaches - traditional as well as innovative. Journal of Documentation, v. 58, n. 4, p. 422- 462, 2002.

MARTINS, A. et al. Uso de uma ontologia de fundamentação para dirimir ambiguidades na modelagem de processos de negócio. In. SIMPÓSIO BRASILEIRO DE SISTEMAS DE INFORMAÇÃO, 7., 2011. Anais... Salvador: UFBA, 2011.

KHOO, C.; NA, J. C. Semantic Relations in Information Science. Annual Review of Information Science and Technology, p. 157-228, 2007.

LUHN, H., Keyword in Context Index for Technical Literature. American Documentation, v. 11, n. 4, p. 288-295, 1960.

MARCONDES, C. H.; CAMPOS, M. L. A. Ontologia e Web Semântica: o espaço da pesquisa em ciência da informação. Ponto de Acesso, v. 2, p. 107-136, jun./jul. 2008.

MARCONDES, C. H; MENDONÇA, M. A. R; MALHEIROS, L. R. Uma proposta de modelo de representação do conhecimento contido no texto de artigos científicos publicados na Web em formato legível por programas. DatagramaZero, v. 7, n. 5, 2006. Disponível em: <http://www.dgz.org.br/out06/Art_03.htm>. Acesso em: 02 de ago. de 2011.

NODINE, M.; FOWLER, J. On the Impact of Ontological Commitment. In: The Workshop on Ontologies In Agent Systems. Proceedings... Bologna: Italy, 2002. Disponível em: <http://ftp.informatik.rwth-aachen.de/Publications/CEUR-WS/Vol-66/oas02-11.pdf>.

Acesso em 14 mai. 2009.

OTLET, Paul. Traité de Documentation: le livre sur le livre. Théorie et pratique. Liège: Centre de Lecture Publique de la Communauté Française de Belgique, 1989. 432p.

PERREAULT, J. Categories and Relators. International Classification, Frankfurt, v. 21, n. 4, p. 189-198, 1994.

POLI, R.; OBRST, L. The Interplay Between Ontology as Categorial Analysis and Ontology as Technology. in R. Poli, M. Healy, A. Kameas, eds., TAO-Theory and Applications of Ontology, Springer, 2009.

RAMALHO, R. A. S. Desenvolvimento e utilização de ontologias em bibliotecas digitais: uma proposta de aplicação. 145 f. Tese (Doutorado em Ciência da Informação) Programa de Pós-Graduação em Ciência da Informação, Universidade Estadual Paulista Júlio de Mesquita Filho - UNESP, Marília, 2010.

RANGANATHAN, S. R. Prolegomena to Library Classification. New York: Asia Publishing House, 1967. 
SALES, L. F. Ontologias de domínio: estudo das relações conceituais e sua aplicação. 139 f. Dissertação (Mestrado em Ciência da Informação) - Convênio IBICT/UFF, Universidade Federal Fluminense, Niterói, 2006.

SHERA, J. H., Kent, A; PERRY, J. W. (ed.). Information systems in documentation. NY: Interscience Publishers, 1957. (Advances in Documentation and Library Science; v. 1).

USCHOLD, M.; GRÜNINGER, M. Ontologies: Principles, Methods, and Applications, Knowledge Engineering Review, v. 1, p. 96-137, 1996.

WILSON, T.D. The Work of the Bristish Classification Research Group. In: WELLISCH, H. (ed.) Subject retrieval in the seventies. Westport: Greeword Publishing Co, 1972. p. 62-71.

\title{
Title
}

The representation of knowledge domains and a theory of representation: the foundational ontology.

\begin{abstract}
The need to provide information in an unambiguous and effective way has motivated the increasing use of ontologies, whose formalism allows greater precision in models of information representation. In this scenario, foundational ontologies have a leading role in defining formally free-domain concepts, and provide a solid theoretical foundation to support the building of domainspecific vocabularies. The understanding of the concepts formalized in foundational ontologies with philosophical basis, must be appropriated by the information scientist, in order to enable him to act in this new scenario, where the representation of information should be directed not only for human understanding, but also for its computational treatment, by using inferences. The purpose of this paper is to present some of these notions contained in the foundational ontology UFO-A, and illustrate, with an example, the semantic differential of a model underpinned by such ontology, in contrast to what could be represented using a thesaurus.
\end{abstract}

\section{Keywords}

Ontologies. Foundational ontologies. Knowledge representation.

\section{Título}

La representación de dominios de conocimientos y una teoría de la representación: la ontología de fundación

\section{Resumen}

La necesidad de proporcionar información en un modo claro y eficaz ha motivado el aumento del uso de ontologías, cuyo formalismo permite una mayor precisión en los modelos de representación de la información. En este escenario, las ontologías de fundación tienen papel destacado en la definición de los conceptos formalmente y independientes de dominio, y proporcionan una sólida base teórica para apoyar la construcción de los vocabularios específicos de dominios. La comprensión de los conceptos formalizados en ontologías de fundación, con base 
en la filosofía, debe ser apropiada por el científico de la información, con el fin de que pueda actuar en este nuevo escenario, donde debe ser la representación de la información dirigida no sólo para el entendimiento humano, sino también para su tratamiento computacional, mediante el uso de inferencias. El objetivo de este trabajo es presentar algunos de estos conceptos contenidos en la ontología de fundación UFO-A, e ilustrar, con un ejemplo, lo diferencial del modelo semántico respaldado por la ontología, frente a la diferencia que existiría caso fuese representada con un tesoro

\section{Palabras clave}

Ontologias. Ontología de Fundación. Representación de Conocimiento.

Recebido em: 07/09/2011

Aceito em: 07/12/2011 